\title{
Geomagnetic dipole moment collapse by convective mixing in the core
}

\author{
Lijun Liu $^{1}$ and Peter Olson ${ }^{2}$ \\ Received 12 March 2009; revised 13 April 2009; accepted 24 April 2009; published 28 May 2009.
}

[1] Convective mixing in the fluid outer core can induce rapid transient decrease of the geomagnetic dipole. Here we determine rates of dipole moment decrease as a function of magnetic Reynolds number following convective instability in a numerical dynamo and in axisymmetric kinematic flows. Our calculations show that mixing flows induce reversed magnetic flux on the core-mantle boundary through expulsion of mostly poloidal magnetic field by convective upwellings. The dipole field collapse is accelerated by enhanced radial diffusion and meridional advection of magnetic flux below the core-mantle boundary. Magnetic energy cascades from the dipole to smaller scales during mixing, producing a filamentary magnetic field structure on the core-mantle boundary. We find that the maximum rate of dipole moment decrease on century time scales is weakly sensitive to the mixing flow pattern but varies with the velocity of the flow approximately as $\mathrm{cRm}^{\beta}$, with $R m$ the magnetic Reynolds number and $(c, \beta) \approx(0.2 \pm 0.07,0.78 \pm$ $0.05)$. According to our calculations, a mixing flow in the outer core with $R m$ in the range of 200-300 can account for the historically-measured rate of decrease of the geomagnetic dipole moment, although it is unlikely that a single mixing flow event with this intensity would cause a full dipole collapse or polarity reversal. Citation: Liu, L., and P. Olson (2009), Geomagnetic dipole moment collapse by convective mixing in the core, Geophys. Res. Lett., 36, L10305, doi:10.1029/ 2009GL038130.

\section{Introduction}

[2] The geomagnetic dipole moment is decreasing at a rate of nearly $6 \%$ per century [Langel et al., 1980; Hulot et al., 2002], which is at least one order of magnitude faster than the free decay rate of the fundamental dipole mode in the core [Olson and Amit, 2006]. This decrease has been sustained for the past 160 years [Jackson et al., 2000]. There is new evidence the axial dipole moment changed relatively little between 1590 and 1840 [Gubbins et al., 2006; Finlay, 2008]. Extensive patches of reversed magnetic flux have evolved on the core-mantle boundary (CMB) over this time [Bloxham and Gubbins, 1985] that have contributed to the observed dipole moment decrease [Gubbins, 1987]. Meanwhile there has been relatively little change in the total magnetic flux crossing the CMB [Benton and Voorhies, 1987].

[3] A variety of mechanisms have been suggested for inducing the reversed magnetic flux patches and producing

\footnotetext{
${ }^{1}$ Seismological Laboratory, California Institute of Technology, Pasadena, California, USA.

${ }^{2}$ Department of Earth and Planetary Sciences, Johns Hopkins University, Baltimore, Maryland, USA.
}

Copyright 2009 by the American Geophysical Union. 0094-8276/09/2009GL038130\$05.00 the dipole moment decrease. These include expulsion of reversed magnetic field across the CMB following resistive instabilities of the toroidal magnetic field inside the core [Bloxham, 1986] possibly related to inner core size [Stanley et al., 2007], and the growth and poleward propagation of kinematic dynamo waves [Gubbins, 1987]. Like resistive instabilities, dynamo wave mechanisms are limited by magnetic diffusion [Gubbins and Gibbons, 2002] and are therefore expected to develop on relatively long time scales in the outer core.

[4] Numerical dynamos reveal there are fast, advectiondriven mechanisms for reducing dipole moment that are induced by fluctuations in the amplitude and pattern of convection. On average, convection in the outer core contributes energy to the dipole field in order to maintain the geodynamo against Ohmic dissipation. But because the convection is time variable, it sometimes has the opposite effect, extracting energy from the dipole field by advective mixing and enhanced magnetic diffusion. These mixing events happen when instabilities reduce the symmetry of the flow [Nishikawa and Kusano, 2008], especially during transition between bistable dynamo states [Simitev and Busse, 2009] and are associated with increases in the amplitude and time-variability of convection [Olson et al., 2009]. Velocity fluctuations in chaotic numerical dynamos are enhanced by the action of the Lorentz force on the fluid, which is heterogeneous in space and time [Christensen and Wicht, 2007].

[5] Several independent lines of evidence indicate that the geodynamo is subject to mixing events. The long-term behavior of the geomagnetic dipole moment is characterized by continuous large-amplitude intensity fluctuations [Valet et al., 2005] including many periods of rapid decrease. On shorter time scales, the pattern of core flow inferred from the geomagnetic secular variation includes significant changes occurring over decades or less [Voorhies, 1995; Holme, 2007; Amit and Olson, 2008; Olsen and Mandea, 2008]. In addition, equatorial symmetry of the core flow in some frozen flux analyses of the secular variation is rather low [Amit and Olson, 2006], which is qualitatively consistent with dynamo model behavior during dipole collapse. Taken together, this evidence suggests that dipole mixing occurs frequently in the outer core and may be active now.

\section{Magnetic Mixing Flows}

[6] We consider the effects of convective instability due to an unstable thermal gradient in a rotating, electrically conducting fluid with an initially axial dipolar magnetic field in the core. This is an idealization of the types of flow variability that occur spontaneously in a continuouslyevolving dynamo. In this case the initial state corresponds to zero flow and free magnetic decay and the convective instability produces flows that result in the mixing event. 

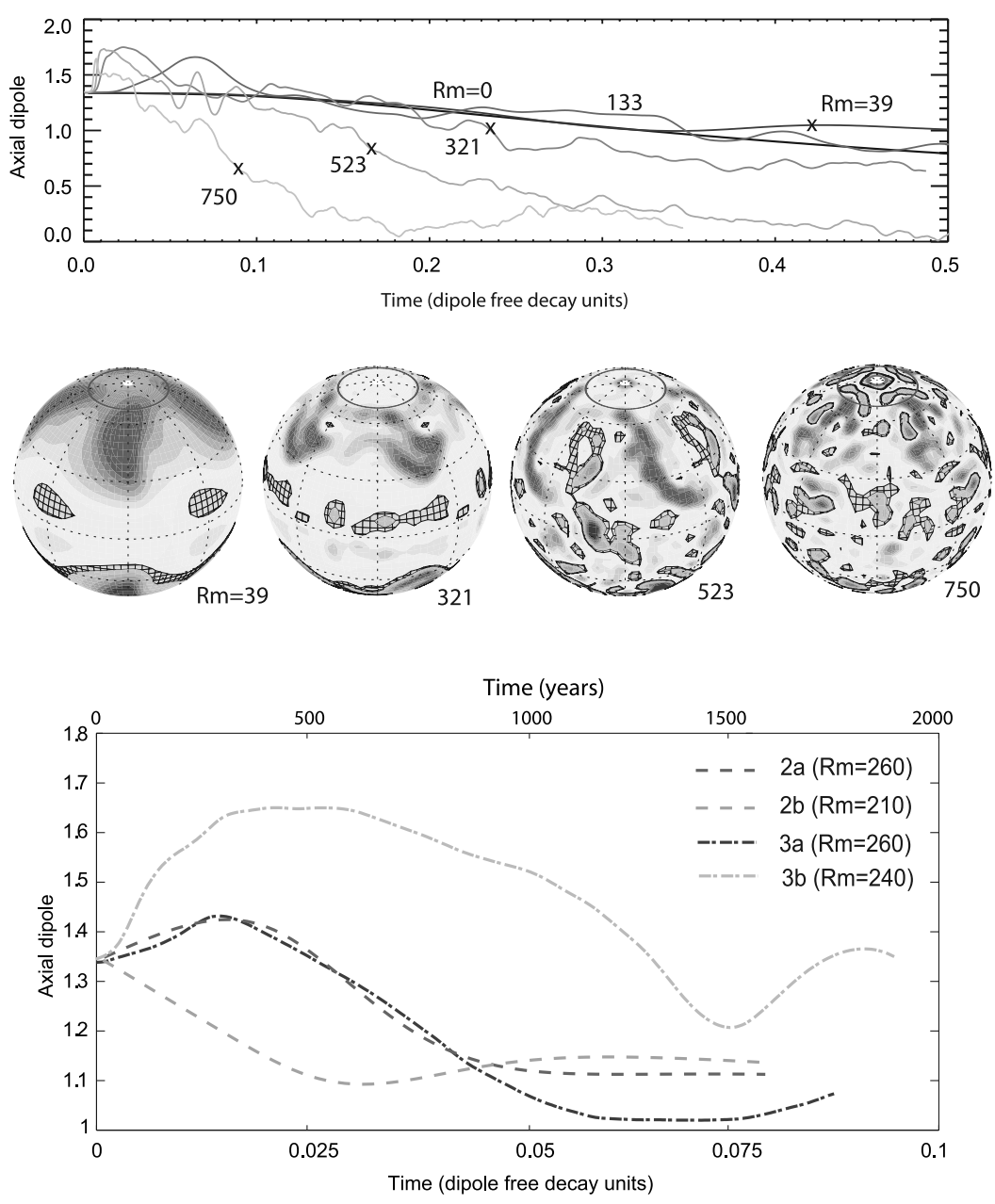

Figure 1. (top) Axial dipole RMS intensity on the core boundary in Elsasser number $(\sqrt{\rho \Omega / \sigma})$ units versus time in dipole decay units following onset of convection in $3 \mathrm{~b}$ dynamo models at Ekman number $E=0.001$ with 4-fold azimuthal symmetry, for various values of $R a$ and $R m$. Cases shown are $R a=3.3 \times 10^{4}, R m=0 ; R a=1 \times 10^{5}, R m=39 ; R a=3.3 \times$ $10^{5}, R m=133 ; R a=1 \times 10^{6}, R m=321 ; R a=2 \times 10^{6}, R m=523$; and $R a=3.3 \times 10^{6}, R m=750$. (middle) Snapshots of radial magnetic field intensity on the CMB in four cases at the times indicated by $\mathrm{x}$ on the time series. Unmeshed, meshed = positive, negative values. (bottom) Axial dipole versus time from four different mixing flows with comparable $\mathrm{Rm}$ values and peak sustained decay rates. The 2D models are axisymmetric kinematic MHD calculations; the 3D models are threedimensional dynamo calculations. Model time is expressed in years assuming $\tau=20 \mathrm{kyr}$.

More realistically, mixing flows involve the instability and transition from one convective flow pattern to another, but in numerical dynamos these spontaneous transitions are typically complex and difficult to reproduce, and are therefore less suitable for systematic analysis.

[7] In order to further reduce the model complexity, we restrict our consideration to well-calibrated types of flows: a three-dimensional dynamo (benchmark \#1 from Christensen et al. [2001]) and for calibration purposes, even simpler twodimensional axisymmetric kinematic flows. For the dynamo models, we consider temperature profiles corresponding to Rayleigh numbers in the range $3.3 \times 10^{4}<R a<5.0 \times 10^{6}$. Two sets of dynamo calculations are discussed, one with no azimuthal symmetry and random initial temperature perturbations (3a-type) the other with prescribed four-fold azimuthal symmetry and a mode four initial temperature perturbations (3b-type). The dynamo calculations were made using the code $M A G$ available at www.geodynamics.org.
[8] For comparison purposes we also made two sets of kinematic MHD calculations in which we prescribe a timeindependent, two-dimensional kinematic flow and compute its effect on an initially dipole-dominant magnetic field. We consider both positive and negative kinematic flows, defined by their direction of motion in the equatorial plane: either with radially-outward motion (2a-type), or with radially inward motion in the equatorial plane (2b-type). The flows also include the geometrical effects of the inner core tangent cylinder with upwellings at polar regions.

[9] Figure 1 shows the axial dipole strength versus time following convective onset in four-fold symmetry dynamos (types 3b) at various magnetic Reynolds numbers $R m$ (based on rms velocity, diffusivity, and shell depth). The case labeled $R m=0$ is subcritical for convection and its dipole decreases approximately like free decay. The $R m=$ 39 case evolves to a steady dynamo, the $R m=133$ case evolves to a dynamo with weak fluctuations, whereas cases 


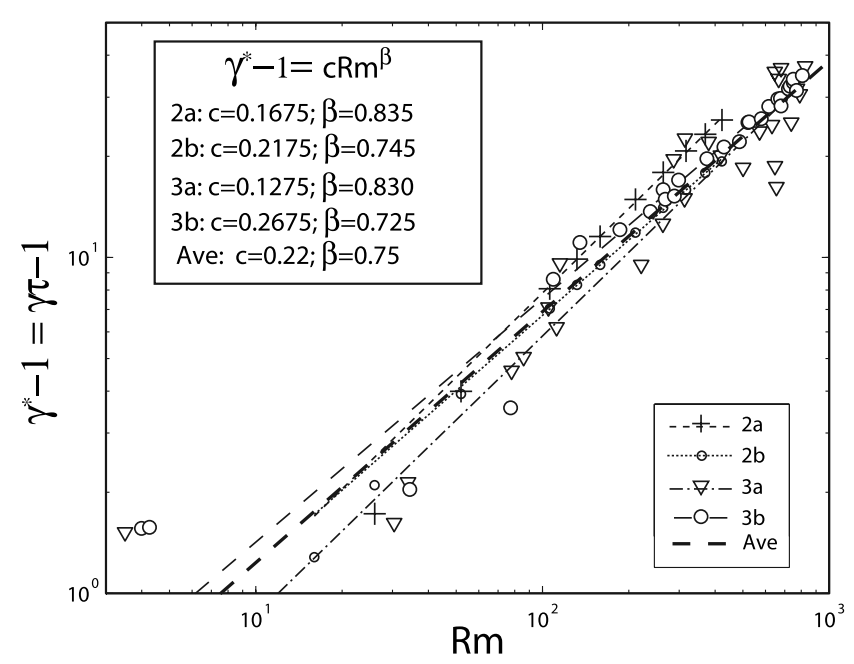

Figure 2. Maximum sustained decay rates (based on $200 \mathrm{yr}$. averages) of dipole field as a function of $R m$ for kinematic and dynamo models. Least squares fits of power laws are shown in the insert. The decay rate is normalized by the dipole free decay rate. Dashed line is composite fit for all data.

with $R m=321,523$, and 750 evolve with strong time variability. In every supercritical case, the dipole field briefly increases, and then undergoes a longer but transient decline, with the average rate and irregularity of this decline increasing with $R m$. Figure 1 also shows snapshots of the radial magnetic field on the core boundary during the transient decay phase for four of these dynamos, arranged in order of increasing $R m$. The concentration of the initial axial dipole field into magnetic filaments - small, highintensity flux spots - increases with $R m$, and in addition, the number and intensity of reversed flux spots increases. The initial, short-lived dipole increase results from poleward advection of the initial axial dipole field by the meridional circulation during convective onset. Longer-lasting dipole decrease is caused by distortion of the axially-symmetric field by convection into fine-scale filaments, the dipole mixing effect, and is more pronounced at larger $\mathrm{Rm}$, where the flow has higher velocity, more time variability, and lower symmetry. We found that cases with $R m>39$, except for $R m=750$, evolved to self-sustaining dynamos that eventually recovered stronger dipole fields. The $R m=750$ case failed as a dynamo, possibly due to inadequate numerical resolution of magnetic filamentation and flux expulsion as reported by Busse and Simitev [2005]. None of the 3b types resulted in polarity reversals within $0.5 \tau$ (approximately $10 \mathrm{kyr}$, with a $\tau \approx 20 \mathrm{kyr}$, free dipole decay time), although this is probably because the imposed 4-fold symmetry acts to suppress reversal processes. Tests using 3a-type dynamos with weaker initial dipoles (not shown) reveal a similar dipole collapse phase and transient reversals, with partial dipole recovery at long times.

[10] The mixing of the internal magnetic fields and flux expulsion processes in types $2 \mathrm{a}$ and $2 \mathrm{~b}$ are similar to those for types $3 b$. Figure 1 (bottom) shows the evolution of the axial dipole versus time for the two axisymmetric kinematic flow types and the two dynamo types at comparable magnetic Reynolds numbers. In type 2a, the equatorial upwelling and the $\mathrm{CMB}$ divergence transports magnetic field lines poleward, producing an initial increase in the axial dipole. However, the dipole collapses shortly thereafter, as the effects of advective mixing of the internal field become dominant. In type $2 b$, the equatorial downwelling and the CMB convergence transport magnetic field lines from higher to lower latitudes, so the dipole collapse begins immediately. In spite of their differences, all the models show approximately the same maximum rates of sustained dipole decrease, although not all at the same times, for the reasons given above. Assuming episodes of maximum sustained decreases in the 3D dynamos are close analogs to the single events in the 2D kinematic cases explains why the magnetic Reynolds number governs the rate of dipole collapse, somewhat independent of the details of the mixing flows.

\section{Dipole Collapse Rates}

[11] In order to determine how the speed of the mixing flow influences the dipole collapse rate, we use the 2D calculations with $R m$-values ranging from 0 to 800 to measure the peak sustained dipole decay rate $\gamma$. Here we define the peak sustained decay rate as the maximum 200-year average rate of dipole decay. For comparison we also measure $\gamma$ in the $3 \mathrm{D}$ dynamo simulations using an averaging interval equal to $1 \%$ of the dipole free decay time. Figure 2 shows normalized peak sustained decay rates, defined by

$$
\gamma^{*}=\gamma \tau
$$

as a function of $R m$ for all four types of calculations. Excluding the low- $R m$ flows, all four types show systematic increase in $\gamma^{*}$ with increasing $R m$ up to $R m \approx 500$. The systematic increase breaks down at larger $R m$-values for the 3a types, probably because the finite numerical resolution is inadequate to capture all the mixing effects of the smallscale flows and magnetic fields in this regime. In addition, their scatter is substantially greater than in the 2D flows or in the dynamos with 4-fold symmetry, reflecting their greater complexity. Nevertheless, the dipole mixing rates of the four types of flow show similar trends. We have fit the normalized dipole decay rates in Figure 2 to power laws

$$
\gamma^{*}-1=c R m^{\beta}
$$

where $c$ and $\beta$ are constants. The factor -1 in (2) accounts for the background decay in the 2D flows. Best fitting values of the constants $c$ and $\beta$ for all four types are shown in insert in Figure 2. All four types fit the power law (2) with coefficients $c$ in the range $0.1275-0.2675$ and with $\beta$ values in the range $0.725-0.835$. An average (composite) fit to all the data is shown by the dashed line in Figure 2 and yields a simple mixing law

$$
\gamma^{*}-1=0.22 R m^{3 / 4}
$$

This average law under-predicts somewhat the mixing rates of the most symmetric flows and over-predicts some of the unconstrained dynamos at high $R m$, However, at intermediate $R m$ the fit is generally good. 

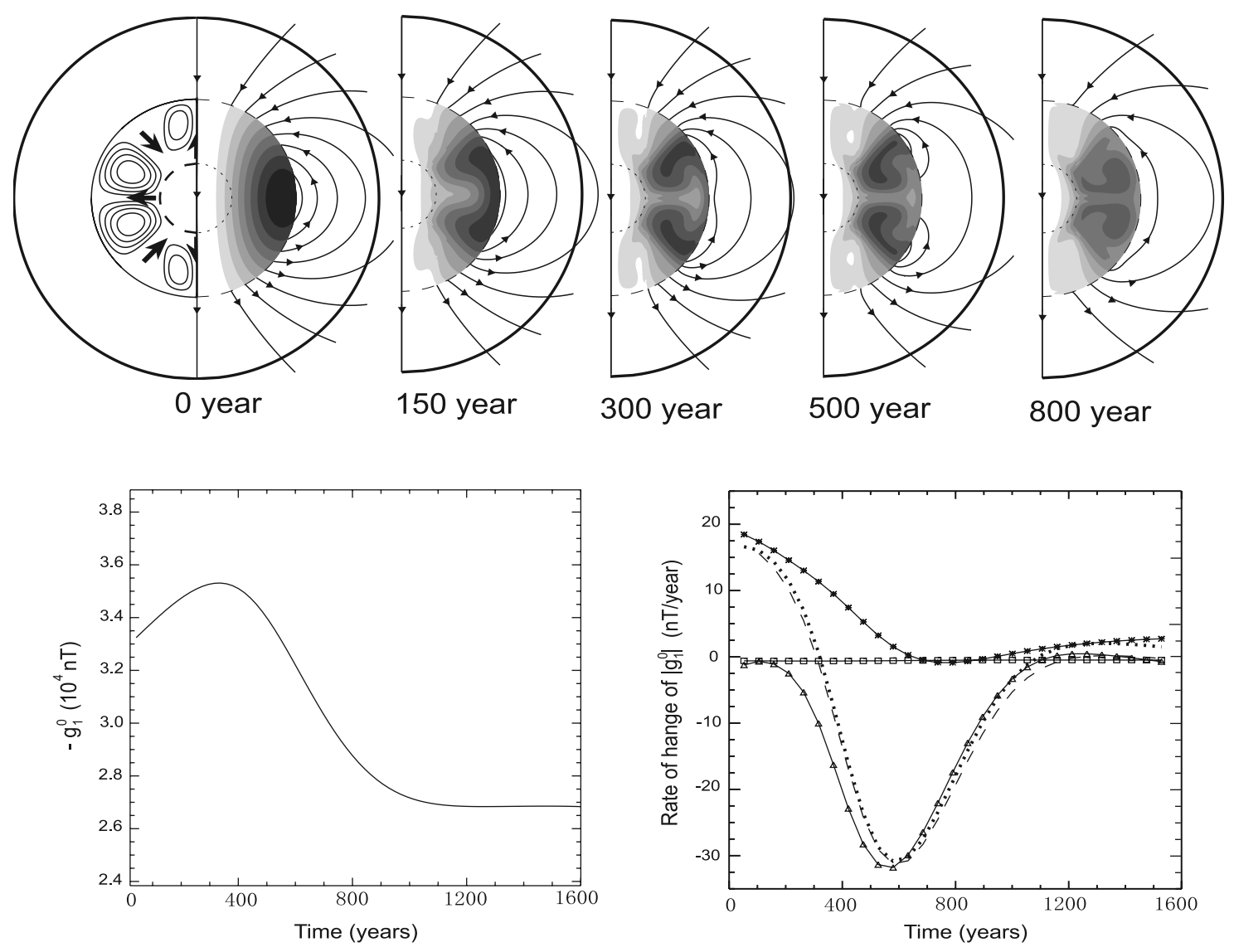

Figure 3. Dipole collapse by axisymmetric mixing flow at $R m=260$. (top) Streamline pattern with magnetic field and internal current density evolution. The outer solid semicircle represents the CMB, and the inner dashed semicircle represents the ICB. Arrows on the streamlines indicate the direction of the fluid velocity. Poloidal magnetic field lines and contours of azimuthal current density are shown on the right. (bottom) The (left) time evolution of $g_{1}^{0}$ and (right) various contributions from (4). Rectangles denote meridional diffusion, triangles denote radial diffusion, and asterisks denote meridional advection. The dotted line is the summation of the three mechanisms, and the dashed line is the rate-of-change of $g_{1}^{0}$ calculated directly.

[12] We can compare (3) with the present-day geomagnetic dipole collapse. The average rate since 1840 corresponds to $\gamma^{*}=\gamma \tau \approx 10$ [Olson and Amit, 2006]. Assuming this value of $\gamma^{*}$ represents the peak sustained rate, the composite fit (3) implies $R m \approx 162$ for the mixing flow in the core. More realistically perhaps, if we use the fit to the fully 3D dynamo models in Figure 2, then we get $R m \approx$ 192. In addition, we must allow for the fact that Figure 2 represents maximum sustained decay rates, whereas the historic rate of geomagnetic dipole decrease may not be its maximum. Accordingly, the appropriate $\mathrm{Rm}$ for presentday mixing in the core could be higher, perhaps as large as 300 or possibly somewhat greater. For comparison, the $R m$ inferred for core flows from frozen flux analyses of the geomagnetic secular variation are in the range 300-500 [Hulot et al., 2002; Amit and Olson, 2006; Holme, 2007]. However, it is likely that the actual $R m$ in the core is larger, because secular variation is weakly sensitive to the component of core flow along magnetic intensity contours, and also because smaller scale fields induced by smaller scale flows in the core are screened by conductivity of the mantle and magnetization of the crust [Jackson and Finlay, 2007]. In any case, mixing flows with Reynolds numbers of order $200-300$ are not unreasonable in the core.

\section{Mixing in the Core Interior and Dipole Collapse on the CMB}

[13] Figure 3 shows the internal mixing effect of type $2 \mathrm{a}$ kinematic flows in time snapshots. The circulation breaks the equatorial electric current torus into smaller tori that diffuse back together over time. The magnetic field is mixed in the center of each meridional cell and concentrates in magnetic flux bundles on the cell margins, as in the classical eddy flux expulsion processes [Weiss, 1966; Proctor and Weiss, 1982] with high and low density of magnetic lines on the CMB indicating fluid downwellings and upwellings, respectively. Reversed flux is expelled at low latitudes, where upwelling drives horizontally magnetized fluid toward the $\mathrm{CMB}$ and induces excess curvature in the field lines. Smaller scale reversed flux forms on the CMB above the edge of strongest mixing eddies, although these reversed patches are short-lived compared to the larger ones. The formation of reversed flux plus their migration toward high latitude induce the rapid dipole moment decrease, which is consistent with the dynamo simulations in Figure 1. 
[14] The axial geomagnetic dipole moment is proportional to the axial dipole Gauss coefficient $g_{1}^{0}$ whose time variation is

$$
\begin{aligned}
\frac{8 \pi R_{o}^{3}}{3} \frac{d g_{1}^{0}}{d t}= & -\int u_{\theta} B_{r} \sin \theta d S-\frac{\lambda}{r} \int \frac{\partial\left(r B_{\theta}\right)}{\partial r} \sin \theta d S \\
& +\frac{\lambda}{r} \int \frac{\partial B_{r}}{\partial \theta} \sin \theta d S
\end{aligned}
$$

where the three terms on the rhs are meridional advection, radial diffusion, and meridional diffusion, respectively. Figure 3 (bottom) shows the total rate-of-change of $g_{1}^{0}$ and the individual contributions from the different terms for type 2a flow with $R m=260$. Among the three mechanisms, meridional diffusion is always negligible, while the other two terms, radial diffusion and meridional advection compete in the decay process. Initially, meridional advection increases the dipole moment, but within a few hundred years time, radial diffusion begins to exert control and the dipole moment begins to decrease rapidly. The effect of radial diffusion diminishes after about 800 years, and the dipole moment stabilizes at about $75 \%$ of its peak value.

\section{Conclusions}

[15] Our calculations show how transient dipole collapse and reversed magnetic flux generation are induced by a mixing flow in the outer core acting on an initially poloidal magnetic field. The transient dipole moment collapse is due to the combined effects of enhanced radial diffusion and meridional advection, with the relative contribution of the two effects depending on the mixing flow pattern. Comparison of kinematic and dynamic calculations indicates that the maximum sustained rate of dipole moment collapse is insensitive to the pattern of the mixing flow, and scales with the flow velocity as the magnetic Reynolds number to a power near $\beta \approx 3 / 4$.

[16] This can be compared with other measures of magnetic field mixing rates. In his study of flux expulsion from closed steady-state eddies in 2D Cartesian geometry, Weiss [1966] finds a time scale for equilibrium that corresponds to an adjustment rate equivalent to $\gamma^{*} \sim R m^{2 / 3}$ in our notation. Tao et al. [1998] parameterize magnetic flux expulsion by small-scale turbulent eddies in terms of an effective magnetic diffusivity that increases with the magnetic Reynolds number of the turbulence like $\lambda_{\text {eff }} \sim \lambda R m$, or in our notation $\lambda_{\text {eff }} \lambda=\gamma^{*} \sim R m$. Accordingly, the exponent in our mixing experiments $\beta \approx 3 / 4$ falls in between the $\beta=2 / 3$ exponent found for steady 2D Cartesian flow, and the $\beta=1$ exponent found for turbulent 2D flow.

[17] Based on our simulations, the rapid decrease of the geomagnetic dipole moment observed during the past 160 years can be produced by a mixing flow in the core with magnetic Reynolds number of order $R m=200-300$. Our results indicate that mixing flows in this range of $R m$ do not result in full dipole collapse or polarity reversals, because the dynamo adjusts to the new flow regime before the dipole field disappears. However, it is possible that full dipole collapse with polarity reversal might follow a closelyspaced sequence of mixing events at this magnetic Reynolds number, or a single mixing event at a much larger magnetic Reynolds number.
[18] Acknowledgment. This research was supported by grant number EAR-0604974 from the Geophysics Program of the National Science Foundation.

\section{References}

Amit, H., and P. Olson (2006), Time-average and time-dependent parts of core flow, Phys Earth Planet Inter., 155, 120-139.

Amit, H., and P. Olson (2008), Geomagnetic dipole tilt changes induced by core flow, Phys. Earth. Planet. Inter, 166, 226-238, doi:10.1016/ j.pepi.2008.01.007.

Benton, E. R., and C. V. Voorhies (1987), Testing recent geomagnetic field models via magnetic flux conservation at the core-mantle boundary, Phys. Earth. Planet. Inter., 48, 350-357.

Bloxham, J. (1986), The expulsion of magnetic flux from the Earth's core, Geophys. J. R. Astron. Soc., 87, 669-678.

Bloxham, J., and D. Gubbins (1985), The secular variation of Earth's magnetic field, Nature, 317, 777-781.

Busse, F. H., and R. Simitev (2005), Convection in rotating spherical fluid shells and its dynamo states, in Fluid Dynamics and Dynamos in Astrophysics and Geophysics, edited by A. M. Soward et al., pp. 359-392, CRC Press, Boca Raton, Fla.

Christensen, U., and J. Wicht (2007), Numerical dynamo simulations, in Treatise on Geophysics, vol. 8, Core Dynamics, edited by G. Schubert, chap. 8, pp. 245-283, Elsevier, Amsterdam.

Christensen, U. R., et al. (2001), A numerical dynamo benchmark, Phys. Earth Planet Inter., 128, 25-34.

Finlay, C. (2008), Historical variation of the geomagnetic axial dipole, Phys. Earth. Planet. Inter., 170, 1-14.

Gubbins, D. (1987), Mechanisms for geomagnetic polarity reversals, Nature, $326,167-169$.

Gubbins, D., and S. J. Gibbons (2002), Three-dimensional dynamo waves in a sphere, Geophys. Astrophys. Fluid Dyn., 96, 481-498.

Gubbins, D., A. L. Jones, and C. Finlay (2006), Fall in Earth's magnetic field is erratic, Science, 321, 900-903.

Holme, R. (2007), Large-scale flow in the core, in Treatise on Geophysics, vol. 8, Core Dynamics, edited by P. Olson, pp. 107-128, Elsevier, Amsterdam.

Hulot, G., C. Eymin, B. Langlais, M. Mandea, and N. Olsen (2002), Smallscale structure of the geodynamo inferred from Oersted and Magsat satellite data, Nature, 416, 620-623.

Jackson, A., and C. C. Finlay (2007), Geomagnetic secular variation and its applications to the core, in Treatise on Geophysics, vol. 5, Geomagnetism, edited by G. Schubert, chap. 5, pp. 148-189, Elsevier, Amsterdam.

Jackson, A., A. R. T. Jonkers, and M. R. Walker (2000), Four centuries of geomagnetic secular variation from historical records, Philos. Trans. R. Soc. London, Ser. A, 358, 957-990.

Langel, R. A., R. H. Estes, G. D. Mead, E. B. Fabiano, and E. R. Lancaster (1980), Initial geomagnetic field model from Magsat vector data, Geophys. Res. Lett., 7, 793-796.

Nishikawa, N., and K. Kusano (2008), Simulation study of symmetrybreaking instability and the dipole field reversal in a rotating spherical shell dynamo, Phys. Plasmas, 15, 082903, doi:10.1063/1.2959120.

Olsen, N., and M. Mandea (2008), Rapidly changing flows in the Earth's core, Nature Geosci., 1, 390-394.

Olson, P., and H. Amit (2006), Changes in Earth's dipole, Naturwissenschaften, 913, 519-542, doi.org/10.1007/s00114-006-0138-6.

Olson, P., P. Driscoll, and H. Amit (2009), Dipole collapse and reversal precursors in a numerical dynamo, Phys. Earth. Planet. Inter., 173, 121-140.

Proctor, M. R. E., and N. O. Weiss (1982), Magnetoconvection, Rep. Prog. Phys., 45, 1317-1379.

Simitev, R. D., and F. H. Busse (2009), Bistability and hysteresis of dipolar dynamos generated by turbulent convection in rotating spherical shells, Earth Planet Lett., 85, 19001, doi:10.1209/0295-5075/85/19001.

Stanley, S., M. T. Zuber, and J. Bloxham (2007), Using reversed magnetic flux spots to determine a planet's inner core size, Geophys. Res. Lett., 34, L19205, doi:10.1029/2007GL030892.

Tao, L., M. R. E. Proctor, and N. O. Weiss (1998), Turbulent flux expulsion, Mon. Not. R. Astron. Soc., 300, 907-914.

Valet, J. P., L. Meynadier, and Y. Guyodo (2005), Geomagnetic dipole strength and reversal rate over the past two million years, Nature, 435, 802-805.

Voorhies, C. V. (1995), Time-varying fluid flow at the top of earth's core derived from definitive geomagnetic reference field models, J. Geophys. Res., 100, 10,029-10,039.

Weiss, N. O. (1966), The expulsion of magnetic flux by eddies, Proc R. Soc London, Ser. A, 293, 1434, 310-328.

L. Liu, Seismological Laboratory, California Institute of Technology, Pasadena, CA 91125, USA. (lijun@gps.caltech.edu)

P. Olson, Department of Earth and Planetary Sciences, Johns Hopkins University, Baltimore, MD 21218, USA. 